\title{
Effectiveness of Telehealth Direct Therapy for Individuals with Autism Spectrum Disorder
}

\author{
Karen Nohelty ${ }^{1}$ (i) $\cdot$ Casey B. Bradford ${ }^{1} \cdot$ Leah Hirschfeld $^{1} \cdot$ CJ Miyake ${ }^{1} \cdot$ Marlena N. Novack $^{1}$
}

Accepted: 26 May 2021 / Published online: 12 July 2021

(C) Association for Behavior Analysis International 2021

\begin{abstract}
The field of applied behavior analysis (ABA) has utilized telehealth for clinical supervision and caregiver guidance with research supporting the use of both modalities. Research demonstrating effectiveness is crucial, as behavior analysts must ensure the services they provide are effective in order to be ethical. With the increased need for patients to access more services via telehealth, due to the novel coronavirus (COVID-19) pandemic, the current study evaluated the efficacy of telehealth direct therapy to teach new skills to individuals with autism spectrum disorder (ASD). This study examined the utility of natural environment teaching and discrete trial training strategies provided over a videoconferencing platform to teach new skills directly to seven individuals with varying ASD severity levels. The targeted skills were taught solely through telehealth direct therapy with varying levels of caregiver support across participants and included skills in the language, adaptive, and social domains. In a multiple baseline design, all seven participants demonstrated mastery and maintenance for all targets; in addition, generalization to family members was assessed for some targets. The evidence suggests that telehealth is a modality that is effective and can be considered for all patients when assessing the appropriate location of treatment.
\end{abstract}

Keywords telehealth direct therapy $\cdot$ discrete trial training $\cdot$ natural environment teaching $\cdot$ generalization

Applied behavior analysis (ABA) is a widely studied and utilized treatment intervention for individuals with autism spectrum disorder (ASD) that has been shown to be effective (Reichow et al., 2018; Smith \& Iadarola, 2015; ViruesOrtega, 2010). ABA-based interventions focus on the treatment of challenging behaviors and the acquisition of skills through concepts like reinforcement, extinction, and prompting. Interventions also utilize training formats such as discrete trial training (DTT) and natural environment teaching (NET). DTT is a method of teaching that involves breaking down skills in a highly structured format and is useful for teaching new behaviors and discriminations (Smith, 2001). These discriminations are taught using a repetitive sequence, including an antecedent, a prompt if needed, a target behavior, and a consequence. In contrast to the structured setting of DTT, NET aims to teach new behaviors in the context of their

Karen Nohelty

k.nohelty@centerforautism.com

1 Center for Autism and Related Disorders, 21600 Oxnard Street, Suite 1800, Woodland Hills, CA 91367, USA relevant environments in contrived or captured situations (Sundberg \& Partington, 1998). Both DTT and NET have their strengths and weaknesses in addressing skill deficits, and both strategies are often included in a patient's treatment program. DTT and NET are considered well-established evidenced-based practices for the treatment of individuals with ASD (Hume et al., 2021; National Autism Center, 2015). These interventions are typically conducted in person by a behavior technician (BT) in the clinic, home, community, or a combination of settings.

The novel coronavirus (COVID-19) pandemic and the need for physical distancing has restricted in-person delivery of medically necessary behavioral health services. In response to the public health emergency declaration, the U.S. Department of Health and Human Services (2020) facilitated the use of telehealth, leading many payors to cover telehealth services for the first time. As opposed to in-person services (i.e., those delivered synchronously with both the patient and clinician in the same location), telehealth services involve the use of technology to provide services when the patient and clinician are apart, either synchronously (e.g., through a videoconferencing platform) or asynchronously (e.g., a clinician reviewing a video or data for a patient, a caregiver accessing 
online content without the presence of a clinician). The Council of Autism Service Providers (2020) asserted that telehealth is a method for delivering health care and not a separate or distinct service. Therefore, the same American Medical Association billing codes, known as Current Procedural Terminology ${ }^{\circledR}$ (CPT; American Medical Association, 2020) codes, are used whether services are provided in person or via telehealth. Types of services that may be provided by qualified clinicians via telehealth include supervision (CPT code 97155; synchronous clinical oversight), direct ABA therapy (CPT code 97153; synchronous adaptive behavior treatment services focused on patient behavior interventions), and caregiver guidance (CPT code 97156; caregiver support and/or direct training on the use of a strategy with synchronous coaching from a clinician, focused on the evaluation of caregiver behavior). This article focuses on telehealth direct therapy regarding the provision of ABA services as provided under the CPT code 97153.

To ensure the continuity of services during the pandemic while maintaining the health and safety of patients and clinicians, it was necessary for providers to shift direct ABA services to telehealth for some patients to continue or supplement therapy, which may involve $25-40 \mathrm{hr}$ per week of intervention (Healy \& Lydon, 2013). Various factors (e.g., prerequisite skills, caregiver availability, childcare arrangements, internet and hardware accessibility) affect how telehealth direct therapy is conducted with each patient. Depending on the patient's level of independence, caregiver support may be needed, and caregivers may assist the BT in various capacities, such as delivering discriminative stimuli $\left(\mathrm{S}^{\mathrm{D}} \mathrm{s}\right)$, prompts, and reinforcement. This service should not be confused with telehealth caregiver guidance. Although caregiver guidance is an important component of an effective ABA program, which supports treatment consistency, generalization, and outcomes (Hong et al., 2018; Postorino et al., 2017), it does not replace direct services led by a BT. Just as caregivers may be involved during in-person direct sessions, they also may be involved in telehealth direct sessions. So long as the focus remains on the patient's skill acquisition, as opposed to the caregiver's performance in delivering the intervention, the services constitute telehealth direct therapy regardless of the caregiver's level of support. Nevertheless, it is recommended that telehealth direct therapy rely on caregiver facilitation in as limited a fashion as possible to promote the patient's independence (Rodriguez, 2020).

Although the rapid shift of services to telehealth was necessary for safety purposes, research on telehealth direct therapy is limited. Although research pertaining to the use of telehealth in the field of ABA treatment for ASD is increasing, this research largely focuses on assessment and caregiver guidance. Prior to the COVID-19 pandemic, the demand for telehealth services as a delivery model for ABA was on the rise due to the growing prevalence of ASD and the shortage of trained clinicians (Yidan Xue Zhang \& Cummings, 2019). The growing body of research on ABA delivered via telehealth is described in two recent systematic reviews. Sutherland et al. (2018) set out to review studies published after 2010 that evaluated the implementation of telehealth for the delivery of assessments, caregiver guidance, or intervention for individuals with ASD. Fourteen studies, which included a combined total 284 participants with ASD ages 19 months to adult, were included in the review. The results of the studies were largely positive and suggested that services implemented via telehealth, including diagnostic and functional behavior assessments, caregiver-implemented services, and caregiver consultation, were equivalent to services delivered in person. In a more recent review, Ferguson et al. (2019) focused more narrowly on ABA intervention, supervision, or caregiver guidance delivered via telehealth for individuals with ASD. Twenty-eight studies were reviewed, which included a total of 307 participants with ASD ages 21 months to 16 years. The outcomes showed positive gains in at least one dependent variable for the participants in $100 \%$ of the reviewed studies and favorable outcomes across all dependent variables in $61 \%$ of the studies. Both systematic reviews included studies that focused on the delivery of assessments and caregiver guidance via telehealth, but neither review included research on the implementation of ABA telehealth therapy directly to the patient.

Although research on telehealth direct therapy is sparse, since the onset of the COVID-19 pandemic more research is emerging on the use of telehealth direct therapy for treating individuals with intellectual and developmental disabilities (Pellegrino \& DiGennaro Reed, 2020) and ASD (Ferguson et al., 2020; Pollard et al., 2020). In a recent study, Ferguson et al. (2020) evaluated the use of telehealth direct therapy to teach comic book characters to six children with ASD. Participants were paired together in dyads, and a DTT intervention was administered using a videoconferencing platform. All participants had reciprocal conversational skills, and none of the participants engaged in aggressive or selfinjurious behaviors. Participants' IQ scores ranged from low (under 70) to average (90-110). Caregiver support was minimal with only a couple participants requiring assistance reentering virtual meetings. Following the intervention, all participants learned primary targets that were directly taught (i.e., the character's name) and secondary targets where participants received instructive feedback but no direct instruction (i.e., the character's superpower). Furthermore, five of the participants acquired the primary and secondary targets of the other participant in their dyad through observation only (i.e., no direct teaching was provided). Skills were maintained at follow-ups conducted up to 9 days posttreatment.

In a recent retrospective analysis, Pollard et al. (2020) reported on the effects of the transition from in-person services to telehealth direct therapy following the 
COVID-19 pandemic. Archival data from 17 clinical cases that transitioned from in-person to telehealth services were examined. The average telehealth dosage was 10.77 hr (range: 5-18). Participants had varying ASD severity levels. Although individual targets were classified as one of three telehealth models based on level of caregiver support, the majority of participants $(n=12)$ received all targets implemented by a BT, either with or without caregiver assistance (e.g., with prompting). The remaining participants $(n=5)$ received caregiverimplemented telehealth model for a portion of their targets. Skill domains targeted in telehealth included language, social, adaptive living, and coping and tolerance skills. Devices used to access telehealth included tablets, smartphones, and computers. An analysis of the continuity of services following the transition to telehealth revealed that participants maintained similar doses of treatment hours per week as compared to their previous inperson services. Furthermore, participants maintained or improved their correct independent responding across all targets.

Although the emerging evidence is promising, more research is needed to evaluate the efficacy of telehealth direct therapy. The extent to which telehealth direct therapy may be used to effectively treat patients with different levels of independence and caregiver support in treatment has not been established. Further research is also needed to determine which types of interventions may be realistically implemented and which skills across developmental domains may be effectively targeted via telehealth. It is critical that telehealth services be effective and ethical (Pollard et al., 2017). In the Professional and Ethical Compliance Code for Behavior Analysts, the Behavior Analyst Certification Board (BACB; 2014) states that behavior analysts should act in the best interests of their client to avoid "interruption or disruption of service" and "make reasonable and timely efforts for facilitating the continuation of behavior-analytic services in the event of unplanned interruptions." The ability for clinicians to provide services via telehealth addresses some ethical considerations for the continuity of services, but more research needs to focus on the effectiveness of telehealth direct therapy. The purpose of the current study was to evaluate the effectiveness of telehealth direct therapy to teach new skills using DTT and NET strategies to individuals with ASD. If found effective, these findings may bolster the evidence base and ethical grounds behind the use of telehealth direct therapy during the COVID-19 pandemic. Furthermore, these findings may help lay the foundation for the use of telehealth direct therapy beyond the current public health crisis to support continuity of services during other unplanned interruptions (e.g., lack of staffing, personal health concerns).

\section{Methods}

\section{Participants and Interventionists}

Study participants received services from the same ABA agency and were located across multiple states (i.e., California, Arizona, Oregon). The following criteria were required for all participants for inclusion in the study: (1) a diagnosis of ASD (American Psychiatric Association, 2013) by a licensed professional (e.g., licensed psychologist, pediatrician, neurologist); and (2) ABA services delivered solely via telehealth. Telehealth direct therapy was provided as the sole service modality due to health concerns related to the COVID-19 pandemic. Prior to receiving telehealth direct therapy, each supervisor considered the following regarding a patient's eligibility for telehealth: (1) whether the patient or their caregivers had access to the internet and a device to participate in telehealth services; (2) whether the patient was able to safely receive telehealth direct therapy without the perceived risk of engagement in severe challenging behaviors that would endanger the patient and others around them; and (3) whether a caregiver was available to assist in the facilitation of therapy sessions to the extent recommended by the primary supervisor. There were no prerequisite skills (e.g., the ability to attend to a screen, discriminate instructions delivered via a screen) required for telehealth direct therapy nor participation in this study. However, a lack of these skills did influence how much caregivers were involved in the implementation of telehealth direct therapy. The Caregiver Support section below discusses the level of caregiver assistance that was needed to implement telehealth direct therapy for each participant. If found to be eligible by the supervisor, telehealth direct therapy was offered as an alternative to clinic-based or home-based sessions in the event that the family was uncomfortable with in-person sessions, given concerns related to COVID-19. Caregivers of all participants provided consent for services to occur via telehealth. After caregivers provided consent, the supervisor modified the program as needed (e.g., behavior intervention plan strategies, skill acquisition plans) to fit the telehealth setting.

Seven participants were included in the study. All participants except one (Jamie) received in-person services prior to the pandemic. See Table 1 for detailed participant characteristics (i.e., age, gender, primary diagnoses, method of ASD diagnosis, severity level, race/ethnicity, primary language). Severity levels were based on the Diagnostic and Statistical Manual of Mental Disorders ( $5^{\text {th }}$ ed.; American Psychiatric Association, 2013) criteria and provided by the supervisor for each participant. The supervisors received training from a licensed psychologist in assigning severity level ratings, which were used to track clinical progress. The severity level data provide information on the participants' current support level needs; although based on the same criteria, they should not be 
Table 1 Participant Characteristics

\begin{tabular}{|c|c|c|c|c|c|c|c|}
\hline Participant & $\begin{array}{l}\text { Age } \\
\text { (years) }\end{array}$ & Gender & $\begin{array}{l}\text { Primary } \\
\text { diagnosis }\end{array}$ & $\begin{array}{l}\text { Method of ASD } \\
\text { diagnosis }\end{array}$ & $\begin{array}{l}\text { Severity level (SC / } \\
\text { RRB) }\end{array}$ & Race / ethnicity & $\begin{array}{l}\text { Primary } \\
\text { language }\end{array}$ \\
\hline Ramon & 4 & Male & ASD & ADOS-2, CARS-2 & $1 / 1$ & Hispanic / Latinx & Spanish \\
\hline Sami & 14 & Male & ASD & ADOS & $2 / 2$ & $\begin{array}{c}\text { Black / African } \\
\text { American }\end{array}$ & English \\
\hline Sergio & 6 & Male & ASD & DSM-5, ADOS-2 & $2 / 2$ & Hispanic / Latinx & Spanish \\
\hline Jackson & 16 & Male & ASD & CARS & $2 / 2$ & Asian & English \\
\hline Zane & 12 & Male & ASD & ADOS-2 & $1 / 1$ & White & English \\
\hline Lara & 4 & Female & ASD & DSM-5 & $3 / 3$ & Asian & English \\
\hline Jamie & 9 & Female & ASD & DSM-5 & $2 / 2$ & White & English \\
\hline
\end{tabular}

Note. $A S D=$ autism spectrum disorder; $\mathrm{SC}=$ social communication; $\mathrm{RRB}=$ restricted, repetitive behaviors; $\mathrm{ADOS}=$ Autism Diagnostic Observation Schedule; CARS $=$ Childhood Autism Rating Scale; DSM = Diagnostic and Statistical Manual of Mental Disorders

confused with diagnostic severity levels, which may only be assigned through a diagnostic evaluation by a clinical psychologist, neurologist, or developmental pediatrician.

Average weekly telehealth therapy hours ranged from 5.4 to 20.9 across participants. Only a proportion of the participants' total telehealth treatment hours were spent addressing the specific targets assessed in this study. See Table 2 for details regarding the intervention for each participant (i.e., method of telehealth access, domain of skills targeted for the study, teaching style, level of caregiver support). ABA programs for all participants were supervised by a Board Certified Behavior Analyst (BCBA) or marriage and family therapist (MFT). The MFT providing case supervision had been working in the field of ABA for over 20 years, attended both BT and supervisor training in $\mathrm{ABA}$ from a large $\mathrm{ABA}$ provider, and was overseen by a BCBA. The supervisor provided individualized direction for each patient's program (e.g., identified target lessons and skills, detailed intervention strategies) via telehealth. The BTs implementing telehealth direct therapy had previous experience providing ABA services in person. In addition, they all received supplemental training in providing telehealth direct therapy prior to the first telehealth session. The training reviewed components such as the structure of a telehealth direct therapy session, caregiver support, and modifications needed due to the telehealth setting (e.g., troubleshooting technical issues, patient engagement modifications). BTs all held a high school diploma; some held additional credentials (e.g., Board Certified Autism Technician, Registered Behavior Technician ${ }^{\circledR}$ [RBT], BCBA). One RBT worked under the MFT; however, she received additional case-specific supervision by a BCBA to meet RBT requirements.

\section{Caregiver Support}

Although a BT directed every session, a caregiver was required to be present in the household and available to provide for the participant's physical care and safety. In addition, caregivers were involved in the session to varying degrees, depending on the needs of the participant. The level of caregiver support required in treatment was determined by the supervisor. Similar to Pollard et al. (2020), three levels of caregiver support were identified for the telehealth direct therapy sessions: technician-delivered telehealth involved the BT providing all interventions (i.e., behavior intervention plan [BIP], reinforcement delivery, prompting, presentation of $\mathrm{S}^{\mathrm{D}} \mathrm{s}$ ); caregiver-assisted telehealth involved BT delivery of all interventions, including presentation of all $\mathrm{S}^{\mathrm{D}} \mathrm{s}$, with caregiver support as needed (e.g., BIP consequence strategy implementation, provision of physical reinforcers, prompting); and

Table 2 Intervention Details

\begin{tabular}{lllll}
\hline Participant & Method of telehealth access & Skill domain & Teaching style & Level of caregiver support \\
\hline Ramon & Laptop / iPad & Language & DTT & Caregiver-assisted \\
Sami & Laptop / iPad & Adaptive & NET & Technician-delivered \\
Sergio & Laptop & Language & DTT & Caregiver-assisted \\
Jackson & Tablet / iPad / laptop & Adaptive & DTT & Technician-delivered \\
Zane & Tablet / iPad & Language & DTT & Technician-delivered \\
Lara & Phone & Language & DTT & Caregiver-implemented \\
Jamie & Desktop / laptop / tablet & Social & NET & Technician-delivered \\
\hline
\end{tabular}

Note. DTT $=$ discrete trial training; $\mathrm{NET}=$ natural environment teaching 
caregiver-implemented telehealth involved the delivery of all interventions by the caregiver upon BT direction. The level of caregiver support in treatment for each participant is detailed in Table 2.

The level of caregiver support in the implementation of telehealth direct therapy differed across participants. Four participants (Sami, Jamie, Jackson, Zane) required no caregiver assistance and received technician-delivered telehealth direct therapy. Prior to the study, they were taught how to independently access and attend telehealth direct therapy sessions. During the study, a caregiver provided assistance, logging the participant into a session only in the event that they were experiencing technological issues.

Two participants required caregiver-assisted telehealth direct therapy (Ramon, Sergio). Prior to the introduction of telehealth direct therapy, caregivers received training in behavior management strategies. During telehealth sessions, caregivers were involved on a minimal basis. Caregivers assisted the participant in logging into Zoom at the start of the session and were always present in the household throughout the session. Implementation of the BIP, delivery of SDs, prompting, and provision of reinforcers were conducted primarily by the BT with caregiver support as needed. For example, if the participant had difficulty orienting towards the BT after a break, the caregiver would verbally prompt the participant to attend to the BT. Also, if the participant logged off the videoconference to escape the session, the caregiver would assist with logging back on and redirect the participant to attend to the session.

One participant, Lara, required caregiver-implemented telehealth direct therapy. During Lara's sessions, the caregiver logged onto the session, prepared all physical materials, and ensured that Lara was present and available for the session. Although the BT interacted with Lara to greet her, build rapport, and elicit her engagement periodically (e.g., sing a song), the caregiver provided all $\mathrm{S}^{\mathrm{D}} \mathrm{s}$, prompts, reinforcers, and BIP strategies upon BT direction. Prior to the study, the caregiver received training to implement all strategies. During the telehealth direct therapy sessions, the BT was responsible for directing the session, which included reviewing past data, determining the current target step, communicating the current step to the caregiver, discussing implementation details, and collecting data. The focus of the session was on provision of therapy to Lara, not on training the caregiver.

The primary focus of this study was on participant responses. Caregivers were provided instructions and given feedback as needed. However, caregiver behaviors were not directly or indirectly measured in this study. The only exception was treatment integrity data for Lara (described below). For the majority of participants, treatment integrity data were collected for the BT delivering the instruction. Because Lara's caregiver delivered the intervention, treatment integrity data were collected on the caregiver. These data were solely collected to demonstrate that the treatment was delivered with fidelity. Any caregiver behavior changes were considered a byproduct of the intervention, and were not within the scope of this study.

\section{Setting and Materials}

For all telehealth direct therapy, a HIPAA-compliant platform (Zoom) was used (Zoom Video Communications Inc., 2016). BTs connected with the participant and their caregiver synchronously. Sessions ranged in duration from 1 to $3 \mathrm{hr}$. Lesson stimuli were either provided over the videoconferencing setting by the BT (e.g., electronically via screen share, physical stimuli held up to the camera) or present in the family's home and provided by the caregiver (i.e., in the case of caregiverimplemented telehealth direct therapy.)

\section{Data Collection and Interobserver Agreement}

To ensure the data were recorded with accuracy, the participant or caregiver were asked to angle the camera towards the participant, so the response could be observed. If the response was missed due to a frozen screen, unclear audio, or the response occurred outside of the screen view, the participant was asked to repeat themselves (for technician-delivered telehealth direct therapy) or the caregiver was consulted as to the correctness of the response (for caregiver-assisted or caregiver-implemented telehealth direct therapy). Data were collected either electronically or, if the data collection device was used to place the zoom call, via pencil and paper and subsequently transferred to the electronic data collection system.

Each participant was assigned three targets for baseline and intervention. These targets were selected by the participant's supervisor and were designated as either DTT or NET targets. Each target selected fell within the following domains: language, adaptive, or social skills.

The dependent variable was the percentage of independent correct responses in a session. Responses were coded as "correct," "prompted-correct," "incorrect," "prompted-incorrect," or "no" response. A correct response was defined as unprompted and meeting the specific response determined by the participant's supervisor. A prompted-correct response was coded if the participant engaged in the predetermined response with any additional cue not part of the $\mathrm{S}^{\mathrm{D}}$. An incorrect response was defined as any unprompted response that did not meet the specific predetermined response criteria. A prompted-incorrect response was coded if the participant did not engage in the predetermined response despite the presence of a prompt. A no response was coded if the participant did not provide any verbal or motor response. Participants were allowed $3-5 \mathrm{~s}$ to initiate a response to an $\mathrm{S}^{\mathrm{D}}$ to receive a score of correct, unless otherwise specified in the lesson instructions 
(i.e., Zane had $10 \mathrm{~s}$ to respond for his targets). Percentage correct for all participants was calculated by dividing the number of correct responses by the total number of trials in the set and multiplying by 100 . Details regarding $\mathrm{S}^{\mathrm{D}} \mathrm{s}$, correct responses, and targets for each participant are included in Table 3.

Interobserver agreement (IOA) data were collected on a minimum of $20 \%$ of all baseline and intervention sessions for all participants. IOA data were collected by the authors during the telehealth direct therapy session or afterwards through video review. IOA was calculated by dividing the total number of agreements by the total of number of agreements plus nonagreements. This number was multiplied by 100 to get the percentage of agreement. The average IOA across participants and conditions was $96.28 \%$ (range: $88.00 \%-100.00 \%$ ). Detailed IOA data by participant and condition are available upon request.

Treatment integrity data were also collected during a minimum of $20 \%$ of all baseline and intervention phases. For technician-delivered and caregiver-assisted telehealth direct therapy sessions, treatment integrity data were collected on the BT. For caregiver-implemented telehealth direct therapy sessions, treatment integrity data were collected on the caregiver. During baseline, each trial consisted of the following steps: present clear $\mathrm{S}^{\mathrm{D}}$ and provide no feedback as to correctness of the response (provide praise, preferred item, or token for general on-task behaviors only). During intervention, each trial consisted of the following steps (as applicable for each trial): present clear $\mathrm{S}^{\mathrm{D}}$; provide praise, preferred item, or token for a correct response; provide informational "no" and repeat the $\mathrm{S}^{\mathrm{D}}$ in response to an incorrect or no response; and implement appropriate prompting procedure. Treatment integrity was calculated by dividing the total number of steps performed correctly by the total number of possible steps and then multiplying by 100 . The average treatment integrity across participants and conditions was $97.42 \%$ (range: 94.37\%-100.00\%). Detailed treatment integrity data by participant and condition are available upon request.

\section{Procedures}

A nonconcurrent multiple baseline across participants design was used. Specific lessons and targets were selected by the supervisor. Lessons fell within the social (i.e., levels of friendship and relationships, prosody), language (i.e., body parts, sequences, functions, manding for information, locations, actions, objects, following instructions), or adaptive (i.e., safety awareness, clothing care, functional typing) skill domains. The supervisor also made the determination as to which teaching style would be used to deliver instruction. Detailed $\mathrm{S}^{\mathrm{D}_{\mathrm{S}}}$ and operational definitions of a correct response for targets for each participant are provided in Table 3 .
Each baseline set was between one and five trials. Neither prompting nor reinforcement was provided for correct responses during the baseline phase. Each intervention dataset was composed of 1-10 trials and included prompting and reinforcement strategies as detailed by the participant's supervisor.

DTT was used for instruction during intervention for five of the participants. Within the DTT format, trials were presented in succession, with a target first presented alone in repetition using errorless learning (most-to-least prompting; Leaf et al., 2020). Mastery criteria set by the supervisor were consistent across participants (i.e., two sessions at $80 \%$ accuracy or higher with first trial correct across two BTs). Once mastery criteria were met, the target was then randomly rotated with one or more previously mastered targets from within the same lesson using error correction (no-no-prompt; Leaf et al., 2020) until the above listed mastery criteria were reached. For one participant, Lara, the supervisor modified procedures based on clinical judgement. Targets were first presented in mass trial with errorless learning. Then, targets were presented in mass trial with error correction. Once mastery criteria were met, instruction proceeded to random rotation. Finally, the target was considered mastered after meeting mastery criteria in random rotation.

Targets were presented within the participant's natural environment, using NET procedures, for two participants (Sami, Jamie). The skills were taught in errorless learning first and, once prompts were faded out, error correction was used (no mastery criteria were set for the transition from errorless learning to error correction). Mastery criteria set by the supervisor were two sessions at $100 \%$ accuracy for Sami and three sessions at $80 \%$ accuracy or higher with first trial correct for Jamie.

Maintenance probes consisted of 1-10 trials of each target and were conducted in the same manner as the intervention (i.e., included the use of prompting and reinforcement) and implemented by the BT with the exception of Lara whose probes were implemented by the caregiver. Maintenance probes were conducted for all participants. Generalization probes were conducted based on participant availability and consisted of one trial of each target. Probes were conducted in the same manner as baseline (i.e., no prompting or reinforcement for correct responses). Generalization probes were implemented in person by the participant's caregiver or sibling and data were collected remotely by the BT during the telehealth session. Maintenance and generalization probes were conducted 1-11 days after the target was mastered.

\section{Results}

A nonconcurrent multiple baseline design was used to assess the effectiveness of telehealth direct therapy. Figure 1 depicts the results for Ramon. All targets were at $0 \%$ throughout 


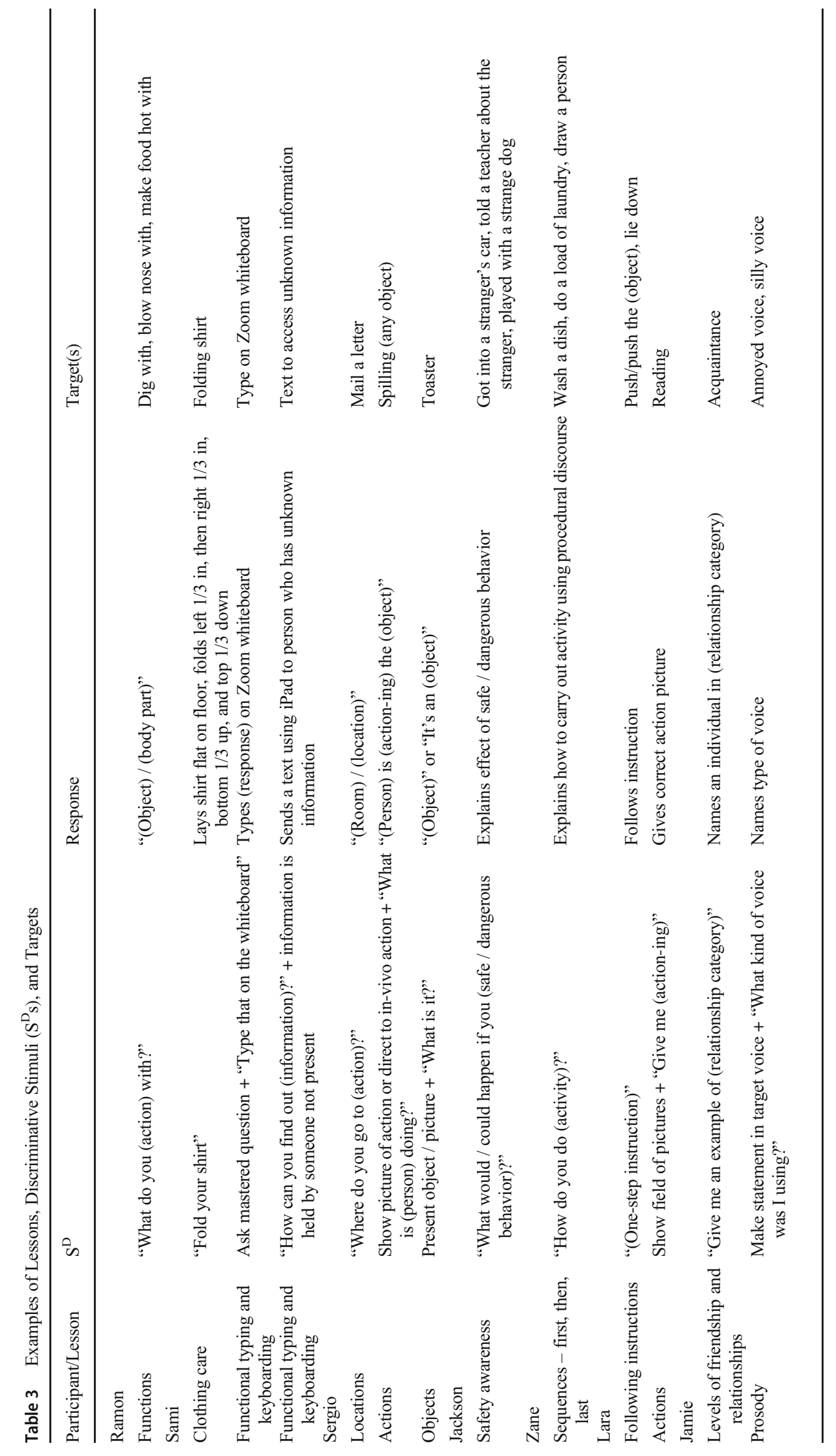


Fig. 1 Percentage of Correct Responses for Ramon During Baseline and Intervention Conditions

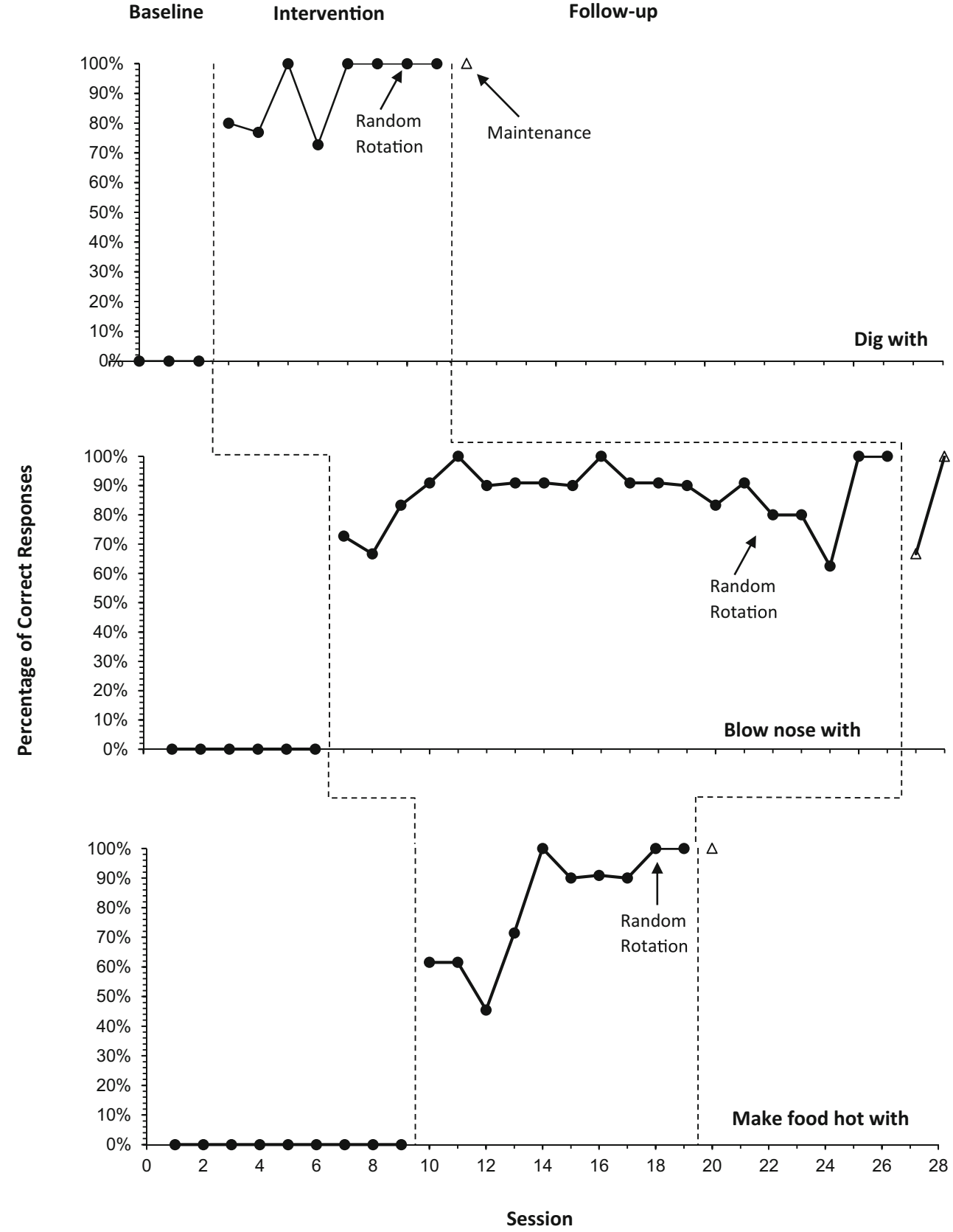

baseline. Upon introduction of the intervention, a clear increase in percentage of correct responses was observed. He mastered the targets "dig with," "blow nose with," and "make food hot with" in 8, 20, and 10 sessions, respectively. Maintenance data for "dig with" and "make food hot with" were both at $100 \%$, whereas maintenance for "blow nose with" dipped to $66.67 \%$ before increasing to $100 \%$. Generalization data were not collected for Ramon.

The results for Sami are displayed in Fig. 2. All baseline data points were at $0 \%$, with an increase in scores within a session of introducing the intervention. "Fold shirt," "type on zoom whiteboard," and "text to access unknown information" were mastered in three, three, and six sessions, respectively.
All maintenance data points were at $100 \%$. Generalization data were not collected for Sami.

Sergio's results are shown in Fig. 3. Baseline data points were at $0 \%$, with an immediate increase in scores when intervention was introduced. The targets "mail a letter," "spilling," and "toaster" met mastery criteria in 6, 14, and 11 sessions, respectively. All maintenance data were at $100 \%$. In a generalization probe conducted by his father, Sergio scored $100 \%$ for all three targets.

Figure 4 displays the results for Jackson. For all targets, baseline data were at $0 \%$. An immediate increase in level was observed upon the introduction of the intervention with the targets "got in his car," "told a teacher," and "strange dog" 
Fig. 2 Percentage of Correct Responses for Sami During Baseline and Intervention Conditions

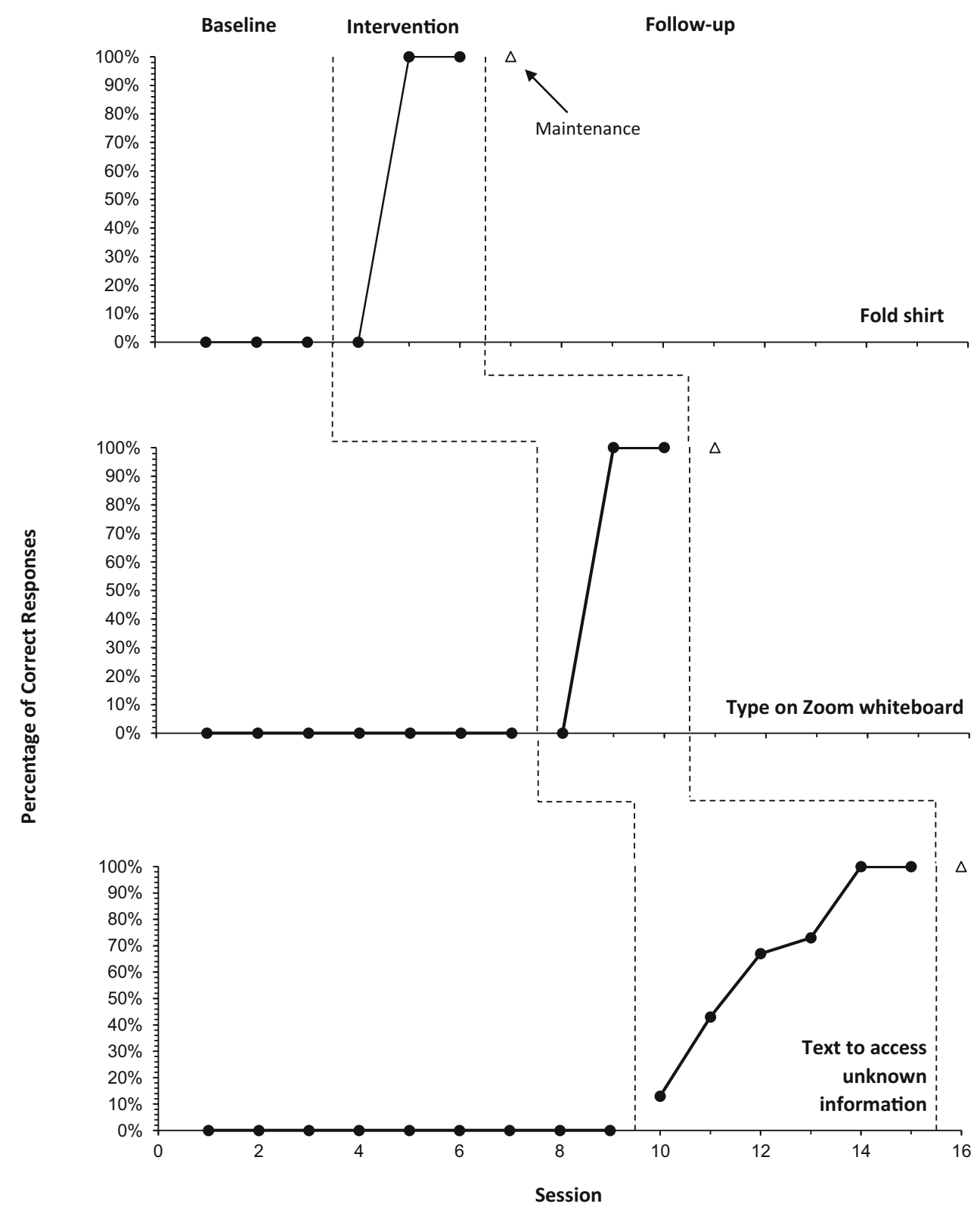

mastered in nine, seven, and nine sessions, respectively. Maintenance data points were all at $100 \%$. In generalization probes conducted by his father, Jackson scored $100 \%$ for all three targets.

Zane's results are shown in Fig. 5. Baseline data were low and stable for all targets. An immediate increase occurred upon the start of intervention with the targets "wash a dish," "do a load of laundry," and "draw a person" mastered within 7, 8, and 10 sessions, respectively. Maintenance data points for Zane were all at $100 \%$. In addition, he scored $100 \%$ for two out of three generalization probes conducted by his father. Although Zane scored $0 \%$ for "do a load of laundry," he only missed the first step in the sequence ("gather clothes") and included all later relevant steps. Thus, the supervisor made the clinical decision to move forward with additional targets for the lesson, coming back to assess generalization later, after study completion.

The results for Lara are depicted in Fig. 6. Baseline data were low and stable for the first and third targets; for the second target, baseline data were moderate but showed a decreasing trend. Following introduction of the intervention, level increased across all three targets, with the targets "push/push the (object)," "reading," and "lie down" mastered in 9,10 , and 17 sessions, respectively. Maintenance probe data were at $100 \%$ for all three targets. Generalization data were not collected for Lara.

The results for Jamie are depicted in Fig. 7. Baseline data were low and stable or decreasing for all targets. Scores increased several sessions following the introduction of intervention for each target, with mastery occurring in 10,21 , and 
Fig. 3 Percentage of Correct Responses for Sergio During Baseline and Intervention Conditions

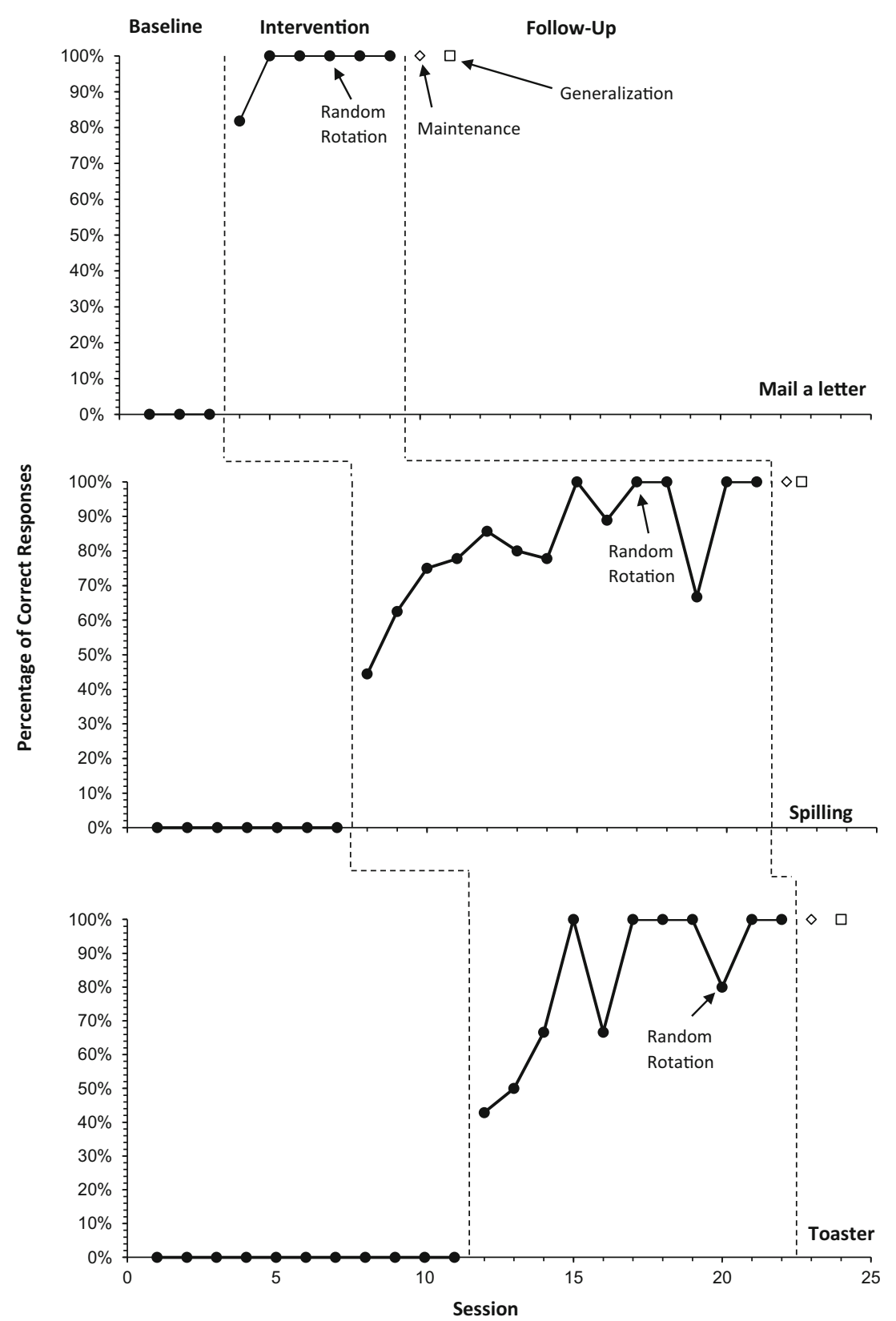

8 sessions for the targets "acquaintance," "annoyed voice," and "silly voice," respectively. Maintenance data for "acquaintance" and "silly voice" were at $100 \%$. Maintenance for "annoyed voice" was at $75 \%$ and then increased to $100 \%$. Generalization data were not collected for Jamie.

\section{Discussion}

The purpose of this study was to evaluate if telehealth direct therapy provided to patients with ASD was effective as measured by skill acquisition. This study expands on the limited body of research on telehealth direct therapy. In line with previous results reported by Ferguson et al. (2020) and Pollard et al. (2020), the current study found telehealth direct therapy to be effective for treating patients with ASD. All participants acquired their target skills to mastery criteria and demonstrated maintenance of skills posttreatment. Furthermore, when assessed, generalization to family members was also shown. This study targeted a wide range of skills (i.e., social, language, adaptive), indicating that telehealth direct therapy can be used to teach skills across domains. Moreover, this study demonstrated that both DTT and NET strategies may be effectively implemented via telehealth.

No prerequisite skills were required for participation in this study and participants with a variety of ASD severity levels 
Fig. 4 Percentage of Correct Responses for Jackson During Baseline and Intervention Conditions

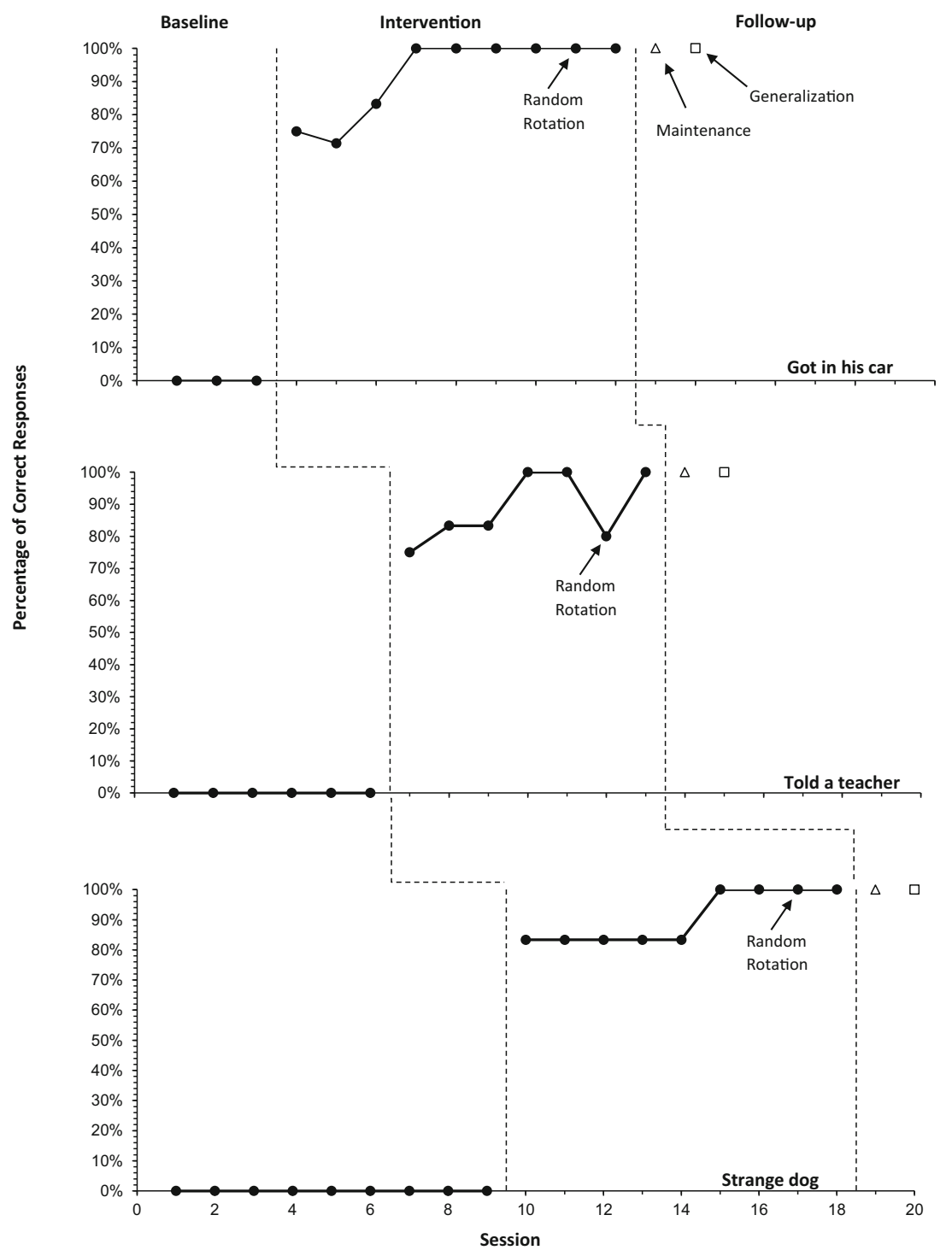

were included. The majority of participants required minimal caregiver support in the implementation of services (six of seven participants). This demonstrates that substantial caregiver support is not required for some patients (e.g., those with appropriate prerequisite skills) to make gains through telehealth direct therapy. It should be noted that the participants who required minimal caregiver support also had severity levels ranging from 1 to 2 . The only participant with severity levels of 3 (Lara) did require caregiver-implemented telehealth direct therapy. This suggests that caregiver implementation may be needed, in particular for patients with high severity levels. The distinction between this service and telehealth caregiver guidance, which has fairly substantial empirical support (Ferguson et al., 2019; Sutherland et al., 2018), is that the session is led by a BT and the focus is on the patient's behavioral change. The current study provides preliminary support for the effectiveness of this service, as covered by the CPT code 97153. Any level of caregiver assistance in telehealth direct therapy may be a challenging obstacle for patients whose caregivers do not have availability to participate in treatment. Nevertheless, this study supports the use of telehealth direct therapy, with appropriate accommodations, for patients with varying severity levels and skillsets.

The current study demonstrated that telehealth direct therapy was not only effective as a modality to provide ABA therapy, but also led to generalization of mastered skills to family members (i.e., caregivers, siblings) in the home setting. Generalization of mastered targets to family members outside of the videoconferencing setting was assessed for four of the seven participants. Generalization was observed for $88.89 \%$ of the targets assessed. In the current study, all targets were taught in the participants' homes. Teaching targets in the participants' everyday environment may have contributed to generalization of the targets to family members. Remote instruction in the home setting may be an added benefit of telehealth direct therapy. 
Fig. 5 Percentage of Correct Responses for Zane During Baseline and Intervention Conditions

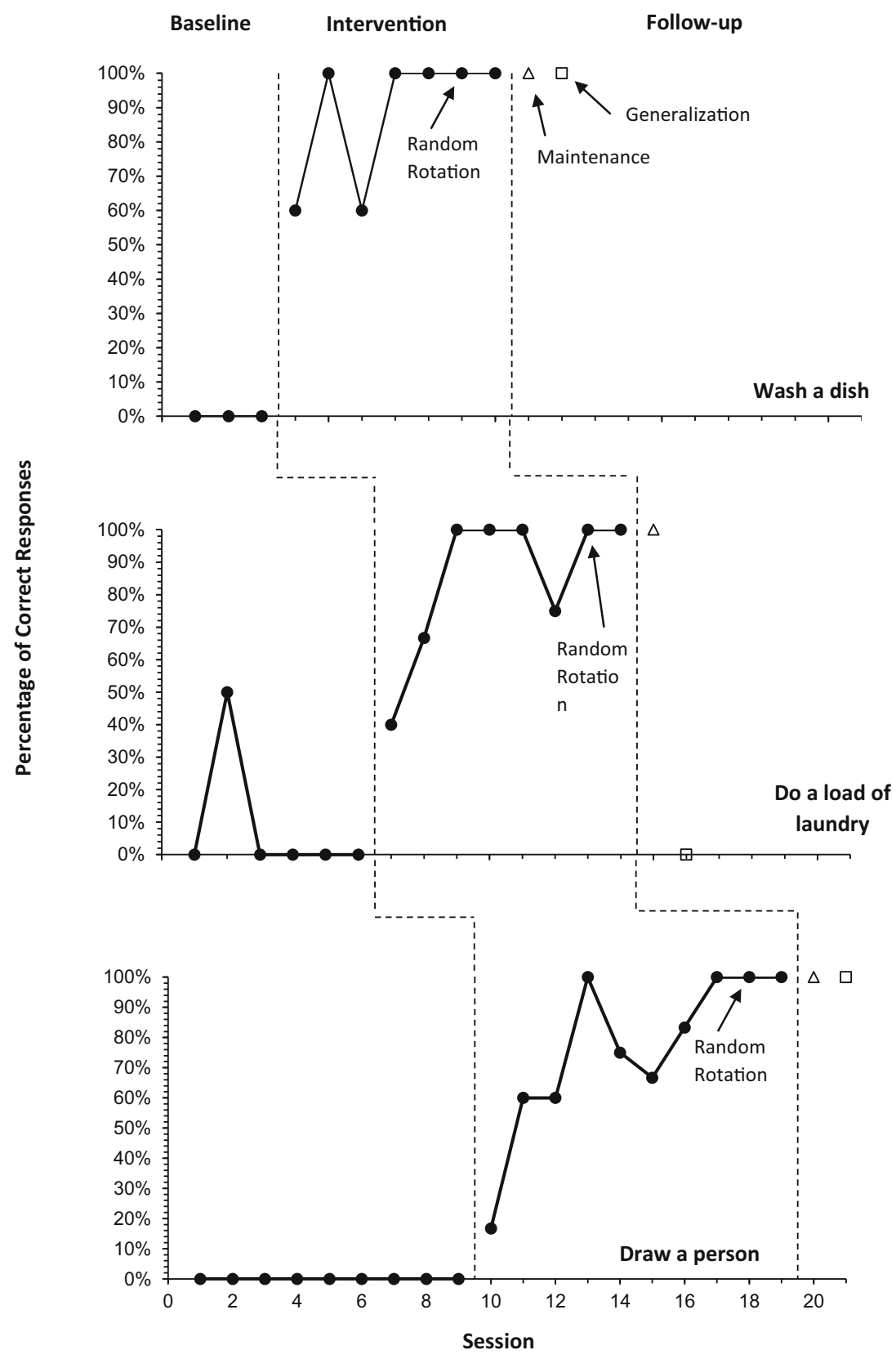

The COVID-19 pandemic is an acute situation affecting the entire world, which has highlighted the utility of telehealth as an emergency measure to provide ABA among other health-care services. As the impact of the COVID-19 pandemic continues to unfold, there may be applications beyond ABA in which fluency in telecommunication technologies would be beneficial for patients. The skills taught through telehealth direct therapy, and the general use of videoconferencing technology, may generalize to other situations (e.g., remote learning for schools and universities, connection to family and friends, vocational skills such as interviews and meetings).
Outside of the current public health crisis, telehealth direct therapy may be an appropriate supplemental or alternative mode to center- or home-based services, in particular in the event that in-person services are not an option due to patientspecific factors (e.g., health concerns, childcare, distance to patient) or staffing factors (e.g., insufficient staff available locally, BT ability to travel). The use of telehealth direct therapy may ensure continuity of services in situations that have traditionally resulted in a gap in services. As telehealth direct therapy can be provided with little caregiver support for some patients, it should be considered a potential clinical setting in ABA treatment programs. Although the current study only 
Fig. 6 Percentage of Correct Responses for Lara During Baseline and Intervention Conditions

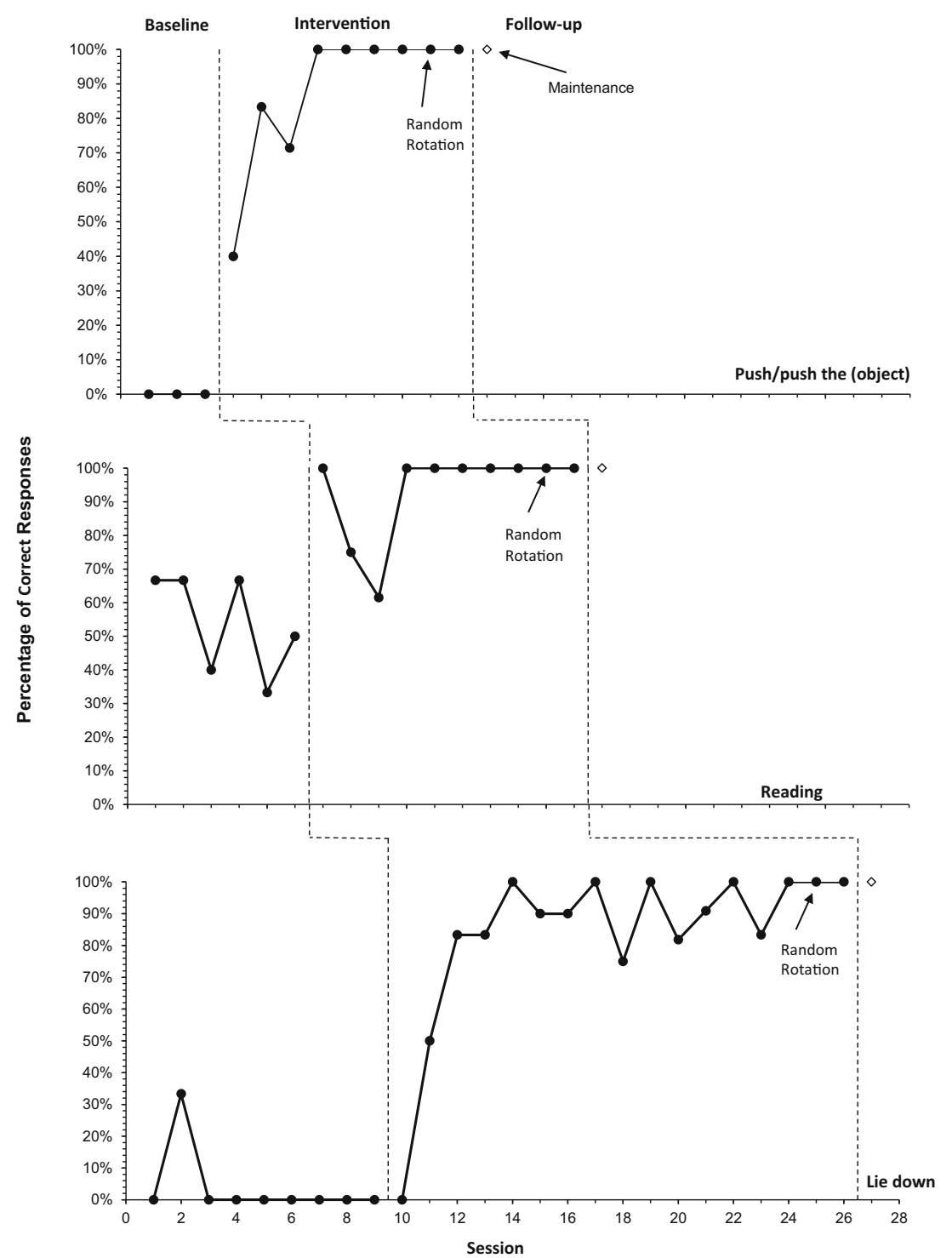

evaluated direct services delivered via telehealth, in other situations it may be appropriate to consider the use of telehealth direct therapy to supplement services delivered in other settings (e.g., home- or center-based services).

Telehealth is also a valuable tool for supporting caregiver participation in treatment. A benefit of telehealth direct therapy is transparency, allowing caregivers easier access to the patient's therapy, including observation of skill acquisition and behavior management strategies implemented in sessions. Telehealth direct therapy may also be a useful option for fading out services and increasingly incorporating caregivers in a natural environment. In addition, for patients that need more support, caregiver-implemented telehealth direct therapy may lead to the generalization of caregiver skills (e.g., strategies to increase appropriate skills and decrease inappropriate skills) outside of the telehealth direct setting, a question that should be assessed in future research.
Although these results are among the first to demonstrate the effectiveness of telehealth direct therapy to individuals with ASD, there were limitations to the study. Although there were several domains taught to participants, several notable developmental domains were not addressed in the current study (i.e., play, executive functions, social cognition, motor skills). Within the domains that were targeted in the study, greater variety in targets was noted for the language and adaptive domains than for the social domain. As target selection was based on clinical judgement by the supervisor, there may be certain domains (e.g., language skills) that are more suitable to teach over videoconferencing than other domains; some skills (e.g., play skills) may be more challenging to target via videoconferencing technologies, which may explain why they were absent from the current study. In addition, it is unclear if telehealth direct therapy would be effective to teach peer interaction. The full extent to which telehealth direct 
Fig. 7 Percentage of Correct Responses for Jamie During Baseline and Intervention Conditions

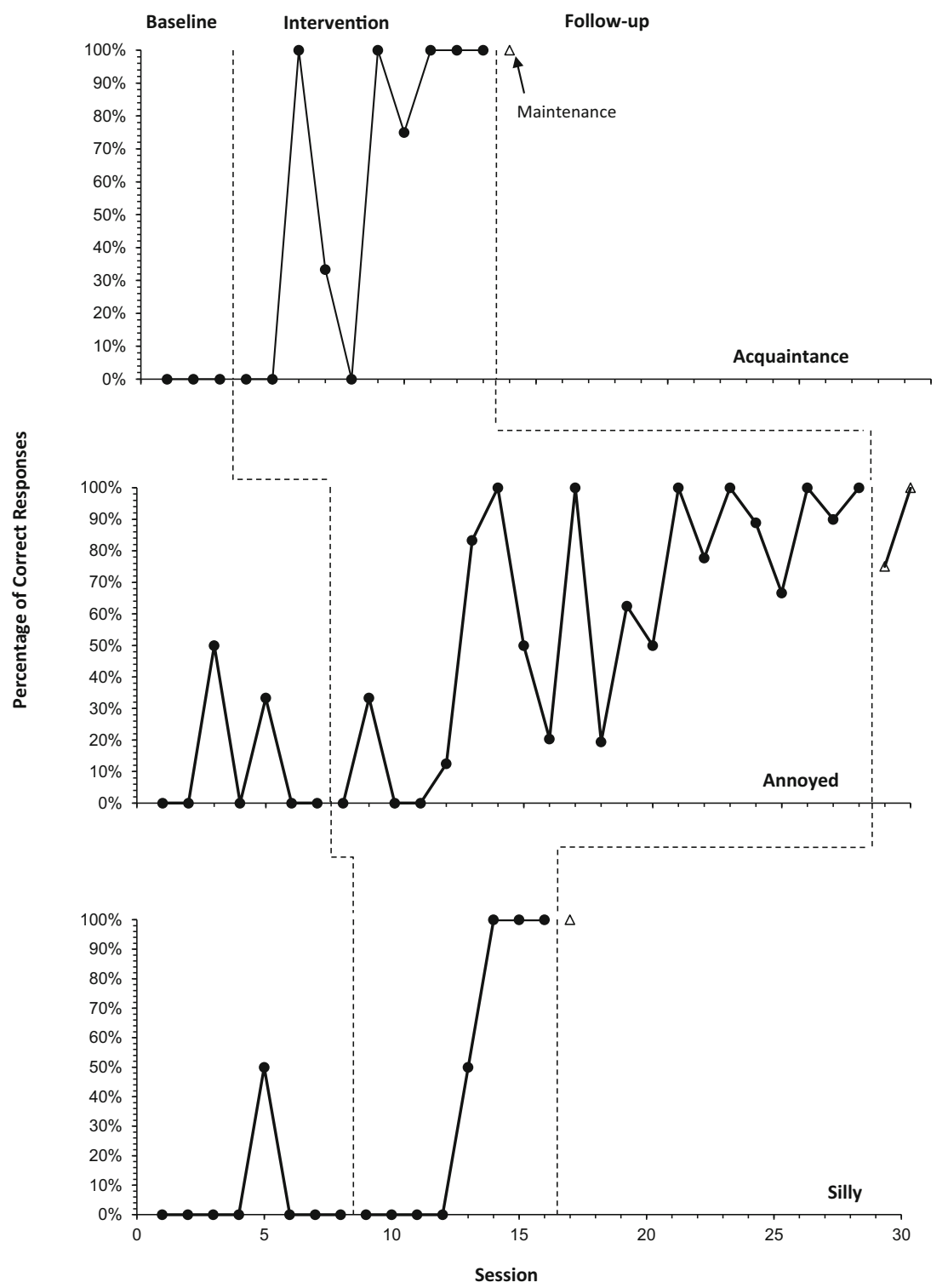

therapy can be effectively used across developmental domains has not been established. Therefore, telehealth direct therapy should be used as one strategy in the broader picture of services to ensure that all appropriate domains are targeted as appropriate for a patient.

There are several additional veins future research can address moving forward. Although this study was completed with a relatively large number of diverse participants, replication of these results is needed to further strengthen the evidence base of ABA delivered in the telehealth setting. In addition, no research has been completed on the use of telehealth direct therapy with adults with ASD. Social validity measures collected from BTs and caregivers would add information regarding the satisfaction with and durability of telehealth direct therapy. Next steps also include assessing telehealth direct therapy with a greater variety of targets in various domains. In particular, studies assessing the use of telehealth direct therapy to teach skills such as play or social cognition would be valuable in determining the efficacy of telehealth direct therapy in those domains. In addition, it would be useful to assess the optimal duration of sessions as it relates to mastery of skills. Future studies could also assess the rate of mastery of skills in patients receiving solely telehealth direct therapy compared to patients receiving solely in-person services. Moreover, as videoconferencing becomes a possible option to keep in touch with family, friends, and peers from school, studies can look at the efficacy of targeting social skills with peer groups over a videoconferencing platform.

The results of this study provide a framework for exploring the use of telehealth as an option for direct therapy, even as centers and in-person services are reestablished following the COVID-19 pandemic. One future possibility is that, when 
making recommendations, clinicians could include the proportion of treatment hours that should take place in each setting (e.g., how many hours to provide in the home, center, community, school, and telehealth settings). Moreover, as future research examines the efficacy of telehealth across skills and domains, clinicians could make determinations about which skills are better targeted using each modality (telehealth vs. in person). Providing treatment across a variety of settings likely has additional benefits to patients, caregivers, and clinicians (e.g., generalization of skills, clinicians understanding a fuller picture of a family's home life), even outside of an emergency situation. Although the extent of telehealth direct therapy's benefits and limitations are still to be determined, this study demonstrates the effectiveness of this model beyond the mere maintenance of skills. Telehealth direct therapy is not only an effective option for ensuring continuity of services during emergencies; telehealth direct therapy is a viable option to supplement in-person services within an ABA treatment program that should be considered by clinicians for all patients.

\section{Declarations}

Ethical Approval All procedures performed in studies involving human participants were in accordance with the ethical standards of the institutional and/or national research committee and with the 1964 Helsinki declaration and its later amendments or comparable ethical standards.

Conflict of Interest The authors declare that they have no conflict of interest.

\section{References}

American Medical Association. (2020). 2021 CPT® professional edition. American Psychiatric Association. (2013). Diagnostic and statistical manual of mental disorders (5th ed.).

Behavior Analyst Certification Board. (2014). Professional and ethical compliance code for behavior analysts. https://www.bacb.com/wpcontent/uploads/BACB-Compliance-Code-english_190318.pdf.

Council of Autism Service Providers. (2020). Practice parameters for telehealth-implementation of applied behavior analysis: Continuity of care during COVID-19 pandemic.

Ferguson, J., Craig, E. A., \& Dounavi, K. (2019). Telehealth as a model for providing behavior analytic interventions to individuals with autism spectrum disorder: A systematic review. Journal of Autism \& Developmental Disorder, 49(2), 582-616. https://doi.org/10. 1007/s10803-018-3724-5.

Ferguson, J. L., Majeski, M. J., McEachin, J., Leaf, R., Cihon, J. H., \& Leaf, J. B. (2020). Evaluating discrete trial teaching with instructive feedback delivered in a dyad arrangement via telehealth. Journal of Applied Behavior Analysis, 53(4), 1876-1888. https://doi.org/10. 1002/jaba.773.

Healy, O., \& Lydon, S. (2013). Early intensive behavioural intervention in autism spectrum disorders. In M. Fitzgerald (Ed.), Recent advances in autism spectrum disorders (Vol. 1; pp. 568-594). IntechOpen. https://doi.org/10.5772/54274.

Hong, E. R., Neely, L., Gerow, S., \& Gann, C. (2018). The effect of caregiver-delivered social-communication interventions on skill generalization and maintenance in ASD. Research in Developmental Disabilities, 74, 57-71. https://doi.org/10.1016/j. ridd.2018.01.006.

Hume, K., Steinbrenner, J. R., Odom, S. L., Morin, K. L., Nowell, S. W., Tomaszewski, B., Szendrey, S., McIntyre, N. S., Yücesoy-Özkan, S., \& Savage, M. N. (2021). Evidence-based practices for children, youth, and young adults with autism: Third generation review. Journal of Autism \& Developmental Disorders, 1-20. https:// doi.org/10.1007/s10803-020-04844-2.

Leaf, J. B., Cihon, J. H., Ferguson, J. L., Milne, C. M., Leaf, R., \& McEachin, J. (2020). Comparing error correction to errorless learning: A randomized clinical trial. Analysis of Verbal Behavior, 36, 120. https://doi.org/10.1007/s40616-019-00124-y.

National Autism Center. (2015). Findings and conclusions: National standards project, phase, 2.

Pellegrino, A. J., \& DiGennaro Reed, F. D. (2020). Using telehealth to teach valued skills to adults with intellectual and developmental disabilities. Journal of Applied Behavior Analysis, 53(3), 12761289. https://doi.org/10.1002/jaba.734.

Pollard, J. S., Karimi, K. A., \& Ficcaglia, M. B. (2017). Ethical considerations in the design and implementation of a telehealth service delivery model. Behavior Analysis: Research \& Practice, 17(4), 298-311. https://doi.org/10.1037/bar0000053.

Pollard, J. S., LeBlanc, L. A., Griffin, C. A., \& Baker, J. M. (2020). The effects of transition to technician-delivered telehealth ABA treatment during the COVID-19 crisis: A preliminary analysis. Journal of Applied Behavior Analysis, 54(1), 87-102. https://doi.org/10. 1002/jaba.803.

Postorino, V., Sharp, W. G., McCracken, C. E., Bearss, K., Burrell, T. L., Evans, A. N., \& Scahill, L. (2017). A systematic review and metaanalysis of parent training for disruptive behavior in children with autism spectrum disorder. Clinical Child \& Family Psychology Review, 20(4), 391-402. https://doi.org/10.1007/s10567-017-02372.

Reichow, B., Hume, K., Barton, E. E., \& Boyd, B. A. (2018). Early intensive behavioral intervention (EIBI) for young children with autism spectrum disorders (ASD). Cochrane Database of Systematic Review, 5. https://doi.org/10.1002/14651858. CD009260.pub2.

Rodriguez, K. (2020). Maintaining treatment integrity in the face of crisis: A treatment selection model for transitioning direct ABA services to telehealth. Behavior Analysis in Practice, 13, 291-298. https://doi. org/10.1007/s40617-020-00429-8.

Smith, T. (2001). Discrete trial training in the treatment of autism. Focus on Autism \& Other Developmental Disabilities, 16(2), 86-92. https://doi.org/10.1177/108835760101600204.

Smith, T., \& Iadarola, S. (2015). Evidence base update for autism spectrum disorder. Journal of Clinical Child \& Adolescent Psychology, 44(6), 897-922. https://doi.org/10.1080/15374416. 2015.1077448 .

Sundberg, M. L., \& Partington, J. W. (1998). Teaching language to children with autism or other developmental disabilities. Behavior Analysts.

Sutherland, R., Trembath, D., \& Roberts, J. (2018). Telehealth and autism: A systematic search and review of the literature. International Journal of Speech-Language Pathology, 20(3), 324 336. https://doi.org/10.1080/17549507.2018.1465123.

U.S. Department of Health \& Human Services. (2020). Telehealth: Delivering care safely during COVID-19. https://www.hhs.gov/ coronavirus/telehealth/index.html 
Virues-Ortega, J. (2010). Applied behavior analytic intervention for autism in early childhood: Meta-analysis, meta-regression and doseresponse meta-analysis of multiple outcomes. Clinical Psychology Review, 30(4), 387-399. https://doi.org/10.1016/j.cpr.2010.01.008.

Yidan Xue Zhang, M. C., \& Cummings, J. R. (2019). Supply of certified applied behavior analysts in the United States: Implications for service delivery for children with autism. Psychiatric Services, 71(4), 385-388. https://doi.org/10.1176/appi.ps.201900058.
Zoom Video Communications Inc. (2016). Security guide. https:// d24cgw3uvb9a9h.cloudfront.net/static/81625/doc/Zoom-SecurityWhite-Paper.pdf

Publisher's Note Springer Nature remains neutral with regard to jurisdictional claims in published maps and institutional affiliations. 